\title{
COMPARATIVE METHODOLOGIES FOR ESTIMATING ON-WATER RESPONSE COSTS FOR MARINE OIL SPILLS
}

\author{
Dagmar Schmidt Etkin \\ Environmental Research Consulting \\ 750 Main Street \\ Winchester, Massachusetts 01890
}

\begin{abstract}
This study reviews three alternative oil spill response cost estimation methodologies as applied to hypothetical spill scenarios in the Gulf of Mexico and San Francisco Bay, California: (1) a model derived from historical data on various spill factors that drive overall cleanup costs; (2) a method using U.S. Area Contingency Plan (ACP) spill scenario plans to estimate costs for mechanical containment and recovery costs to be extrapolated to other hypothetical spill scenarios; and (3) a method that estimates the labor and equipment required for mechanical containment and recovery operations and the resulting costs. A method for estimating dispersant costs is also discussed. The easy-to-use model derived from historical data is shown to be a good cost estimation tool.
\end{abstract}

\section{Cleanup cost estimation methods}

Cleanup cost factor model based on historical spill data. Historical oil spill cost data collected by Environmental Research Consulting and studies conducted by a number of other researchers have shown that per-unit (per-gallon or per-ton) oil spill cleanup costs vary considerably by: location (particularly with regard to shoreline proximity and national jurisdiction), oil type, spill size, and cleanup methodology employed (Allen and Ferek, 1993; Etkin, 1998a, b, c; Harper et al., 1995; Moller et al., 1987; Monnier, 1994; Peck et al., 1996).

Previous papers by this author (Etkin, 1999, 2000) have examined the relationships between a number of factors to determine a more precise per-unit cleanup cost for a particular oil spill scenario. In the latter study, rudimentary formulae were derived:

$$
\mathrm{C}_{u i}=\mathrm{C}_{l i} \mathrm{t}_{i} \mathrm{o}_{i} \mathrm{~m}_{i} \mathrm{~s}_{i} ; \mathrm{C}_{l i}=\mathrm{r}_{i} \mathrm{l}_{i} \mathrm{C}_{n} ; \text { and } \mathrm{C}_{\mathrm{e} i}=\mathrm{C}_{\mathrm{u} i} \mathrm{~A}_{i}
$$
where $\mathrm{C}_{\mathrm{u} i}$ is response cost per unit for scenario, $i ; \mathrm{C}_{l i}$ is cost per unit spilled for scenario, $I ; \mathrm{C}_{n}$ is general cost per unit spilled in nation, $n ; \mathrm{C}_{\mathrm{e} i}$ is estimated total response cost for scenario, $I$; $\mathrm{t}_{i}$ is oil type modifier factor for scenario, $i ; \mathrm{o}_{i}$ is shoreline oiling modifier factor for scenario, $i ; \mathrm{m}_{i}$ is cleanup methodology factor for scenario, $i ; \mathrm{s}_{i}$ is spill size modifier factor for scenario, $i ; \mathrm{r}_{i}$ is regional location modifier factor for scenario, $i$; $1_{i}$ is local location modifier for scenario, $i ; \mathrm{A}_{i}$ is specified spill amount for scenario, $i$.

The modifiers for the various factors are shown in Tables 1 and 2. The algorithm and modifiers were derived from analyses of over 200 oil spill case studies, which revealed that:

- Per-unit costs were higher for spills involving more persistent oils.

- Per-unit costs were highest for responses relying on mechanical and manual methods.
- Per-unit costs were higher for smaller spills.

- Per-unit costs were higher for nearshore and port spills than for offshore spills.

- Per-unit costs were higher for spills involving extensive shoreline oiling.

- Per-unit costs varied by region and were highest for Asia, followed by the United States.

Shoreline cleanup operations are indirectly covered since the model presents cleanup costs as a whole, but relative degree shoreline oiling is one of the modifying factors. For spill scenarios with extensive shoreline impact, shoreline cleanup operation costs can be estimated by assuming that $80-90 \%$ of total costs are for shoreline cleanup (based on Etkin 1998b, c; Unpublished research project, P. Franken, University of Arizona, 1994). Conversely, to isolate mechanical recovery costs, one can estimate that $10-20 \%$ of the total spill response costs are for these operations.

Area Contingency Plan spill scenario mechanical response operation cost extrapolations. Area Contingency Plans (ACPs) in the United States often contain hypothetical spill scenarios, which outline the resources and strategies that would be required in a response. The responses described for each scenario generally cover the most immediate aspects of mechanical containment and recovery operations, and do not allude to dispersant application operations or shoreline cleanup operations. A survey of $\mathrm{ACPs}{ }^{1}$ revealed a wide spectrum of spill scenarios ranging from nonexistent (or not yet entered) to fairly detailed descriptions of response strategy.

Scenario response strategies were used to estimate cleanup response costs for other hypothetical spill scenarios by extrapolation for spill amount regardless of other factors, such as oil type (Figure 1). This involved taking described equipment and labor allocations and calculating costs based on average U.S. Coast Guard (USCG) district-specific equipment and labor costs in USCG Basic Ordering Agreements (BOAs) as presented in Etkin (1998b) converted into 1999 U.S. dollar values. The labor costs take into account different worker types and respective pay scales (regular, premium, and overtime work) in different regions as reflected by USCG district.

The limitations to this approach include the fact that the ACP scenarios tend to be very specific in terms of location and oil type and to involve relatively small spill sizes, making it difficult to relate them to hypothetical situations. For example, a 5,000-gallon No. 2 fuel spill could not easily be projected to a 10 milliongallon crude oil spill even for the site. In addition, ACPs rarely stipulate the estimated time to complete mechanical operations. 
Table 1. Average per-unit marine oil spill cleanup costs by nation/region in 1999 U.S. dollars.

\begin{tabular}{|c|c|c|}
\hline Country & US\$/gallon & US\$/ton \\
\hline \multicolumn{3}{|l|}{ North America } \\
\hline Canada & 22.14 & $6,508.14$ \\
\hline United States & 87.13 & $25,614.63$ \\
\hline Average & 67.41 & $19,814.63$ \\
\hline \multicolumn{3}{|l|}{ Latin America } \\
\hline Argentina & 7.87 & $2,316.61$ \\
\hline Brazil & 19.04 & $5,600.72$ \\
\hline Chile & 3.10 & 910.42 \\
\hline Mexico & 2.88 & 850.32 \\
\hline St. Kitts/Nevis & 10.48 & $3,085.81$ \\
\hline Uruguay & 11.47 & $3,368.25$ \\
\hline Venezuela & 40.20 & $1,817.83$ \\
\hline Average & 10.41 & $3,055.76$ \\
\hline \multicolumn{3}{|l|}{ Africa } \\
\hline Egypt & 15.06 & $4,428.90$ \\
\hline Morocco & 32.89 & $9,675.07$ \\
\hline Mozambique & 0.04 & 6.09 \\
\hline Nigeria & 6.02 & $1,766.75$ \\
\hline South Africa & 9.92 & $2,917.54$ \\
\hline Average & 10.75 & $3,163.93$ \\
\hline \multicolumn{3}{|l|}{ Europe } \\
\hline Denmark & 38.04 & $11,180.41$ \\
\hline Estonia & 23.20 & $6,820.62$ \\
\hline Finland & 7.19 & $2,115.29$ \\
\hline France & 7.83 & $2,301.58$ \\
\hline Germany & 36.41 & $10,702.67$ \\
\hline Greece & 29.03 & $8,530.29$ \\
\hline Ireland & 16.35 & $4,807.49$ \\
\hline Italy & 22.26 & $6,541.19$ \\
\hline Latvia & 31.34 & $9,212.35$ \\
\hline Lithuania & 0.26 & 78.12 \\
\hline Netherlands & 22.63 & $6,655.37$ \\
\hline Norway & 78.61 & $23,118.08$ \\
\hline Spain & 1.48 & 438.68 \\
\hline Sweden & 53.22 & $15,642.36$ \\
\hline United Kingdom & 10.48 & $3,082.80$ \\
\hline Yugoslavia & 5.15 & $1,541.40$ \\
\hline Average & 36.75 & $10,807.83$ \\
\hline \multicolumn{3}{|l|}{ South Pacific } \\
\hline Australia & 20.36 & $5,991.33$ \\
\hline New Zealand & 9.50 & $2,791.35$ \\
\hline Average & 19.38 & $5,698.88$ \\
\hline \multicolumn{3}{|l|}{ Middle East } \\
\hline Israel & 7.87 & $2,313.60$ \\
\hline UAE & 2.16 & 636.99 \\
\hline Average & 3.60 & $1,057.50$ \\
\hline \multicolumn{3}{|l|}{ Asia } \\
\hline Hong Kong & 15.14 & $4,452.94$ \\
\hline Japan & 117.75 & $34,619.92$ \\
\hline Malaysia & 260.90 & $76,589.29$ \\
\hline Philippines & 2.31 & 676.51 \\
\hline Singapore & 1.32 & 390.61 \\
\hline South Korea & 43.60 & $12,814.96$ \\
\hline Average & 93.53 & $27,495.83$ \\
\hline
\end{tabular}

Note: Derived from Etkin (2000).
Table 2. Cleanup cost factor modifiers.

\begin{tabular}{ll}
\hline Cost factor & Modifier \\
\hline Oil type & \\
No. 2 fuel (diesel) & 0.18 \\
Light crude & 0.32 \\
No. 4 fuel, No. 5 fuel & 1.82 \\
Crude & 0.55 \\
Heavy crude & 0.65 \\
No. 6 fuel & 0.71 \\
Spill size & \\
$<10,000$ gal $(<34 \mathrm{t})$ & 2.00 \\
$10,000-100,000$ gal $(34-340 \mathrm{t})$ & 0.65 \\
$100,000-500,000 \mathrm{~g}(340-1,700 \mathrm{t})$ & 0.27 \\
$500,000-1,000,000$ gal $(1,700-3,400 \mathrm{t})$ & 0.15 \\
$1-10$ million gal $(3,400-34,000 \mathrm{t})$ & 0.05 \\
(>10 million gal $(>34,000 \mathrm{t})$ & 0.01 \\
Location type & \\
Nearshore & 1.46 \\
In port & 1.28 \\
Offshore & 0.46 \\
Primary method cleanup & \\
Dispersants & 0.46 \\
In situ burning & 0.25 \\
Mechanical & 0.92 \\
Manual & 1.89 \\
Natural cleansing & 0.10 \\
Shoreline oiling & \\
$0-1$ km & 0.47 \\
2-15 km & 0.54 \\
20-90 km & 0.61 \\
100 km 500 km & 1.06 \\
\hline & 1.53 \\
\hline
\end{tabular}

Note: Derived from Etkin (2000).

Cost estimations based on labor/equipment requirements with modifications. A modified approach involved the study of ACP scenarios and the Response Plan Equipment Caps Review (USCG, 1999), along with reviews of historical case studies to estimate the effort required-worker-days and equipment required for on-water mechanical containment and recovery operations. Again, labor and equipment costs were based on USCG BOAs.

The work estimations were coupled with information on the general behavior of different oil types and amounts using the National Oceanic and Atmospheric Administration's (NOAA) Automated Data Inquiry for Oil Spills (ADIOS) software. The oil behavior data were used to modify the estimated response work estimates for slick spread, dispersion, evaporation, and emulsification by the time mechanical operations were underway.

The following assumptions (based on Michel and Cotsapas, 1997) were applied:

- Fifty percent of floating oil could be recovered or attempted to be recovered.

- Dispersed and/or evaporated oil could not be recovered by mechanical recovery techniques.

- Emulsification increased oily liquid volume by four. No. 2 fuel oil would not emulsify. 


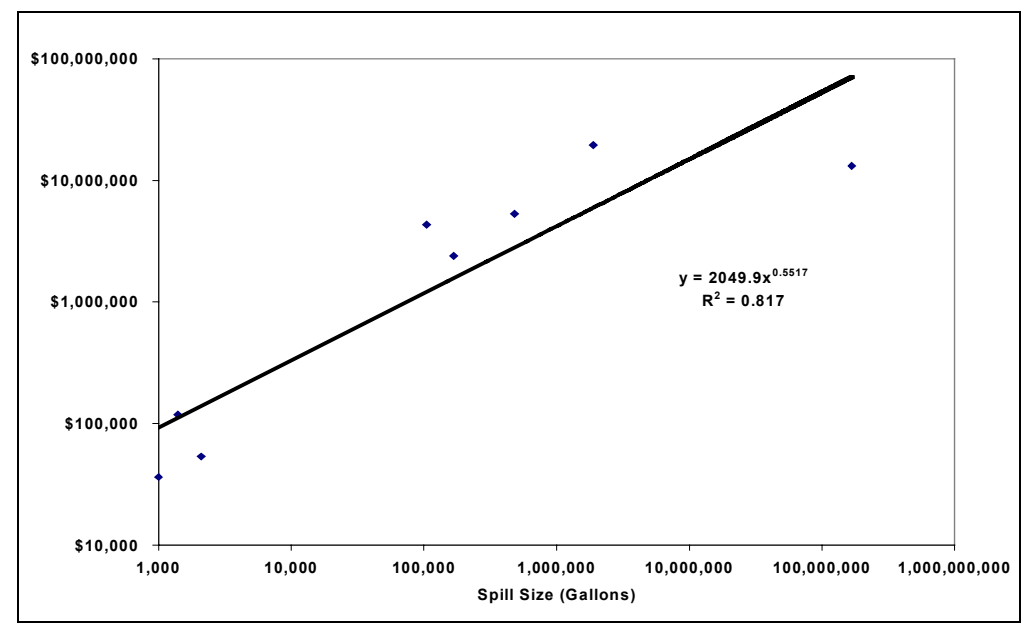

Figure 1. Mechanical recovery costs for ACP scenarios in 1999 U.S. dollars (excluding disposal, shoreline, and equipment decontamination costs).

- Costs for shore-based support for skimming systems were $12 \%$ of on-water costs.

- Helicopter overflights (one for spills under 500,000 gallons; two for larger spills) were charged for 12-hour days for the entire time oil was present on water surface.

- Cleanup crews worked for 12-hour workdays.

- Wages were paid as $67 \%$ straight wages, $20 \%$ premium wages, and $13 \%$ overtime wages.

- Crews consisted of $1 \%$ project managers, $3 \%$ supervisors, $67 \%$ skilled laborers, and $29 \%$ unskilled laborers.

- Costs for labor/equipment increased annually as per the average U.S. Consumer Price Index.

- Skimming capacity was $20 \%$ of nameplate capacity (effective daily recovery capacity [EDRC]) to account for various environmental conditions, as per USCG mandates.
- $\quad$ Spilled oil spread as described in the tables in Tables 3 and 4.

The estimation methods were applied to hypothetical spill scenarios involving two different oil types (No. 2 fuel and crude oil) in two locations (Gulf of Mexico off Galveston Bay, Texas and San Francisco Bay, California) for five different spill amounts $(10,000 \mathrm{gal} / 34 \mathrm{t} ; 100,000 \mathrm{gal} / 340 \mathrm{t} ; 500,000 \mathrm{gal} / 1,700 \mathrm{t} ; 5$ million gal/17,000 t; 10 million gallons/34,000 t).

Dispersant application costs. Cost estimations for dispersant applications were not possible using the ACPs since dispersant use was not described in any of the plans examined. Dispersant use could be stipulated in the historical data model to provide a response cost estimate when dispersants are used as the primary response strategy. This method was applied to the hypothetical spill scenarios.

Table 3. Estimation of spill amount from slick size.

\begin{tabular}{|c|c|}
\hline Spill size (gal) & $\begin{array}{l}\text { Estimated fresh slick size } \\
\text { for } 0.1 \mathrm{~mm} \text {-thick slicks }\left(\text { mile }^{2}\right)^{1}\end{array}$ \\
\hline 10,000 & 0.98 \\
\hline 100,000 & 9.80 \\
\hline 500,000 & 49.02 \\
\hline $5,000,000$ & 490.20 \\
\hline $10,000,000$ & $2,450.98$ \\
\hline
\end{tabular}

${ }^{1}$ Assuming continuous spreading without containment. Source: Etkin (1999c).

Table 4. Estimated slick size for specified spill scenario sizes.

\begin{tabular}{|c|c|c|c|c|c|}
\hline Type of oil & Appearance & $\begin{array}{l}\text { Thickness } \\
\text { (mm) }\end{array}$ & $\begin{array}{l}\text { Volume } \\
\left(\mathrm{m}^{3} / \mathrm{km}^{2}\right)\end{array}$ & $\begin{array}{l}\text { Volume } \\
\left(\mathrm{gal} / \mathbf{k m}^{2}\right)\end{array}$ & $\begin{array}{l}\text { Volume } \\
\text { (gal/mile }^{2} \text { ) }\end{array}$ \\
\hline Fresh & Black-dark brown & $0.1^{1}$ & 100 & 26,420 & 10,200 \\
\hline Sheen & Rainbow & 0.0003 & 0.3 & 80 & 207 \\
\hline Sheen & Silvery & 0.0001 & 0.1 & 26 & 16 \\
\hline Mousse ( $60 \%$ water) & Frothy brown/orange & $>1$ & $>1,000^{2}$ & $105,000^{2}$ & $271,950^{2}$ \\
\hline
\end{tabular}




\section{Results of application to hypothetical spill scenarios}

Cost breakdowns for the equipment/labor estimation method are shown in Tables 5 through 8 . The results of the cost estimates for mechanical operations for the scenarios are shown in Table 9 through 12. Graphic comparisons of the different mechanical recovery operation cost estimation methods for the Galveston Bay scenarios are shown in Figures 2 and 3.

Table 5. Gulf of Mexico/Galveston Bay No. 2 fuel spill scenarios estimated costs for mechanical recovery based on mechanical recovery estimations.

\begin{tabular}{|c|c|c|c|c|}
\hline Spill amount (gal) & Equipment costs (\$) & Labor costs (\$) & Additional costs (\$) & Total costs ${ }^{1}(\$)$ \\
\hline 10,000 & 11,430 & 18,210 & $\begin{array}{l}\text { Federal: } 4,000 \\
\text { State: } 3,500 \\
\text { Disposal: } 3,404 \\
\text { Decont.: } 2,000\end{array}$ & $\begin{array}{l}42,544 \\
(37,140)\end{array}$ \\
\hline 100,000 & 231,532 & 225,201 & $\begin{array}{l}\text { Federal: } 125,783 \\
\text { State: } 28,189 \\
\text { Mgt. Team: } 50,400 \\
\text { Disposal: } 8,800 \\
\text { Decont.: } 10,000\end{array}$ & $\begin{array}{l}679,905 \\
(661,105)\end{array}$ \\
\hline 500,000 & $1,519,114$ & 774,627 & $\begin{array}{l}\text { Federal: } 503,132 \\
\text { State: } 112,756 \\
\text { Mgt. Team: } 201,600 \\
\text { Disposal: } 74,000 \\
\text { Decont.: } 25,000\end{array}$ & $\begin{array}{l}3,210,229 \\
(3,111,229)\end{array}$ \\
\hline $5,000,000$ & $5,160,081$ & $2,989,919$ & $\begin{array}{l}\text { Federal: } 754,698 \\
\text { State: } 169,134 \\
\text { Mgt. Team: } 302,400 \\
\text { Disposal: } 2,664,000 \\
\text { Decont.: } 167,500\end{array}$ & $\begin{array}{l}12,207,732 \\
(9,376,232)\end{array}$ \\
\hline $10,000,000$ & $8,657,790$ & $4,839,463$ & $\begin{array}{l}\text { Federal: } 754,698 \\
\text { State: } 169,134 \\
\text { Mgt. Team: } 302,400 \\
\text { Disposal: } 5,772,000 \\
\text { Decont.: } 410,000\end{array}$ & $\begin{array}{l}20,905,485 \\
(14,723,485)\end{array}$ \\
\hline
\end{tabular}

\footnotetext{
${ }^{\mathrm{T}}$ Total costs w/o disposal, equipment decontamination.
}

Table 6. Gulf of Mexico/Galveston Bay crude oil spill scenarios estimated costs for mechanical recovery based on mechanical recovery estimations.

\begin{tabular}{|c|c|c|c|c|}
\hline Spill amount (gal) & Equipment costs $(\$)$ & Labor costs $(\$)$ & Additional costs (\$) & Total costs $^{1}(\$)$ \\
\hline \multirow[t]{5}{*}{10,000} & 65,396 & 31,471 & Federal: 10,000 & 157,783 \\
\hline & & & State: 5,000 & $(128,867)$ \\
\hline & & & Mgt. Team: 2,000 & \\
\hline & & & Disposal: 9,916 & \\
\hline & & & Decont.: 19,000 & \\
\hline \multirow[t]{5}{*}{100,000} & 847,694 & 369,853 & Federal: 20,000 & $1,603,747$ \\
\hline & & & State: 10,000 & $(1,317,547)$ \\
\hline & & & Mgt. Team: 70,000 & \\
\hline & & & Disposal: 96,200 & \\
\hline & & & Decont.:190,000 & \\
\hline \multirow[t]{5}{*}{500,000} & $4,438,779$ & $1,307,493$ & Federal:754,698 & $8,477,504$ \\
\hline & & & State: 169,134 & $(6,792,504)$ \\
\hline & & & Mgt. Team: 302,400 & \\
\hline & & & Disposal: 555,000 & \\
\hline & & & Decont.:950,000 & \\
\hline \multirow[t]{5}{*}{$5,000,000$} & $14,991,872$ & $5,279,837$ & Federal: $1,257,830$ & $36,288,429$ \\
\hline & & & State: 281,890 & $(21,312,429)$ \\
\hline & & & Mgt. Team: 504,000 & \\
\hline & & & Disposal: $5,476,000$ & \\
\hline & & & Decont.:9,500,000 & \\
\hline \multirow[t]{5}{*}{$10,000,000$} & $27,012,809$ & $10,757,507$ & Federal: $1,509,396$ & $70,026,780$ \\
\hline & & & State: 338,268 & $(40,222,780$ \\
\hline & & & Mgt. Team: 604,800 & \\
\hline & & & Disposal: $10,804,000$ & \\
\hline & & & Decont.: $19,000,000$ & \\
\hline
\end{tabular}

\footnotetext{
${ }^{1}$ Total costs w/o disposal, equipment decontamination.
} 
Table 7. San Francisco Bay No. 2 fuel spill scenarios estimated costs for mechanical recovery based on mechanical recovery estimations.

\begin{tabular}{|c|c|c|c|c|}
\hline Spill amount (gal) & Equipment costs (\$) & Labor costs (\$) & Additional costs (\$) & Total costs ${ }^{1}(\$)$ \\
\hline 10,000 & 11,430 & 34,034 & $\begin{array}{l}\text { Federal: } 4,000 \\
\text { State: } 3,500 \\
\text { Disposal: } 3,404 \\
\text { Decont.: } 2,000\end{array}$ & $\begin{array}{l}58,368 \\
(52,964)\end{array}$ \\
\hline 100,000 & 231,532 & 437,959 & $\begin{array}{l}\text { Federal: } 125,783 \\
\text { State: } 28,189 \\
\text { Mgt. Team: } 50,400 \\
\text { Disposal: } 8,800 \\
\text { Decont.: } 10,000\end{array}$ & $\begin{array}{l}892,663 \\
(873,863)\end{array}$ \\
\hline 500,000 & $1,519,114$ & $1,510,414$ & $\begin{array}{l}\text { Federal: } 503,132 \\
\text { State: } 112,756 \\
\text { Mgt. Team: } 201,600 \\
\text { Disposal: } 74,000 \\
\text { Decont.: } 25,000\end{array}$ & $\begin{array}{l}3,946,016 \\
(3,847,016)\end{array}$ \\
\hline $5,000,000$ & $5,160,081$ & $5,830,675$ & $\begin{array}{l}\text { Federal: } 754,698 \\
\text { State: } 169,134 \\
\text { Mgt. Team: } 302,400 \\
\text { Disposal: } 2,664,000 \\
\text { Decont.: } 167,500\end{array}$ & $\begin{array}{l}15,048,488 \\
(12,216,988)\end{array}$ \\
\hline $10,000,000$ & $8,657,790$ & $9,492,014$ & $\begin{array}{l}\text { Federal: } 754,698 \\
\text { State: } 169,134 \\
\text { Mgt. Team: } 302,400 \\
\text { Disposal: } 5,772,000 \\
\text { Decont.: } 410,000\end{array}$ & $\begin{array}{l}25,558,036 \\
(19,376,036)\end{array}$ \\
\hline
\end{tabular}

${ }^{1}$ Total costs w/o disposal, equipment decontamination.

Table 8. San Francisco Bay crude spill scenarios estimated costs for mechanical recovery based on mechanical recovery estimations.

\begin{tabular}{|c|c|c|c|c|}
\hline Spill amount (gal) & Equipment costs (\$) & Labor costs (\$) & Additional costs (\$) & Total costs ${ }^{1}(\$)$ \\
\hline 10,000 & 70,486 & 59,330 & $\begin{array}{l}\text { Federal: } 10,000 \\
\text { State: } 5,000 \\
\text { Mgt. Team: } 2,000 \\
\text { Disposal: } 9,916 \\
\text { Decont.: } 19,000\end{array}$ & $\begin{array}{l}190,732 \\
(161,816)\end{array}$ \\
\hline 100,000 & 696,998 & 721,723 & $\begin{array}{l}\text { Federal: } 20,000 \\
\text { State: } 10,000 \\
\text { Mgt. Team: } 70,000 \\
\text { Disposal: } 96,200 \\
\text { Decont.:190,000 }\end{array}$ & $\begin{array}{l}1,804,921 \\
(1,518,721)\end{array}$ \\
\hline 500,000 & $3,755,456$ & $2,555,370$ & $\begin{array}{l}\text { Federal: } 754,698 \\
\text { State: } 169,134 \\
\text { Mgt. Team: } 302,400 \\
\text { Disposal: } 555,000 \\
\text { Decont.: } 950,000\end{array}$ & $\begin{array}{l}9,042,058 \\
(7,537,058)\end{array}$ \\
\hline $5,000,000$ & $12,882,128$ & $10,318,493$ & $\begin{array}{l}\text { Federal: } 1,257,830 \\
\text { State: } 281,890 \\
\text { Mgt. Team: } 504,000 \\
\text { Disposal: } 5,476,000 \\
\text { Decont.:9,500,000 }\end{array}$ & $\begin{array}{l}39,217,341 \\
(24,241,341)\end{array}$ \\
\hline $10,000,000$ & $23,637,219$ & $21,147,115$ & $\begin{array}{l}\text { Federal: } 1,509,396 \\
\text { State: } 338,268 \\
\text { Mgt. Team: } 604,800 \\
\text { Disposal: } 10,804,000 \\
\text { Decont.: } 19,000,000\end{array}$ & $\begin{array}{l}77,040,798 \\
(47,236,798)\end{array}$ \\
\hline
\end{tabular}


Table 9. Estimated mechanical recovery operation costs for Gulf of Mexico off Galveston Bay No. 2 fuel oil scenarios in 1999 U.S. dollars.

\begin{tabular}{cccc}
\hline Spill amount (gal) & $\begin{array}{l}\text { ACP } \\
\text { extrapolation } \mathbf{( \$ )}\end{array}$ & $\begin{array}{l}\text { Mechanical recovery } \\
\text { modeling (\$) }\end{array}$ & $\begin{array}{c}\text { Etkin historical cost } \\
\text { data model (\$) }\end{array}$ \\
\hline 10,000 & 330,014 & 37,140 & 270,000 \\
100,000 & $1,175,527$ & 661,105 & 970,000 \\
500,000 & $2,856,633$ & $3,111,229$ & $4,300,000$ \\
$5,000,000$ & $10,175,469$ & $9,376,232$ & $10,200,000$ \\
$10,000,000$ & $14,915,323$ & $14,723,485$ & $20,400,000$ \\
\hline
\end{tabular}

${ }^{1}$ Excludes disposal, shoreline cleanup, and decontamination costs.

${ }^{2}$ Includes manual shoreline cleanup.

Table 10. Estimated mechanical recovery operation costs for Gulf of Mexico off Galveston Bay crude oil scenarios 1999 U.S. dollars.

\begin{tabular}{cccc}
\hline Spill amount (gal) & $\begin{array}{l}\text { ACP extrapolation } \\
\mathbf{( \$ )}^{\mathbf{1}}\end{array}$ & $\begin{array}{l}\text { Mechanical recovery } \\
\text { modeling (\$) }\end{array}$ & $\begin{array}{c}\text { Etkin historical cost } \\
\text { data model (\$) }\end{array}$ \\
\hline 10,000 & 330,014 & 128,867 & 915,000 \\
100,000 & $1,175,527$ & $1,317,547$ & $5,000,000$ \\
500,000 & $2,856,633$ & $6,792,504$ & $25,000,000$ \\
$5,000,000$ & $10,175,469$ & $21,312,429$ & $110,000,000$ \\
$10,000,000$ & $14,915,323$ & $40,222,780$ & $300,000,000$ \\
\hline
\end{tabular}

${ }^{1}$ Excludes disposal, shoreline cleanup, and decontamination costs.

${ }^{2}$ Includes manual shoreline cleanup.

Table 11. Estimated mechanical recovery operation costs for San Francisco Bay No. 2 fuel oil scenarios 1999 U.S. dollars.

\begin{tabular}{crrr}
\hline Spill amount (gal) & $\begin{array}{l}\text { ACP extrapolation } \\
\mathbf{( \$ )}^{\mathbf{1}}\end{array}$ & $\begin{array}{l}\text { Mechanical recovery } \\
\text { modeling } \mathbf{( \$ ) ^ { \mathbf { 1 } }}\end{array}$ & $\begin{array}{c}\text { Etkin historical cost } \\
\text { data model (\$) }\end{array}$ \\
\hline 10,000 & 330,014 & 52,964 & 350,000 \\
100,000 & $1,175,527$ & 873,863 & $2,400,000$ \\
500,000 & $2,856,633$ & $3,847,016$ & $8,900,000$ \\
$5,000,000$ & $10,175,469$ & $12,216,988$ & $16,000,000$ \\
$10,000,000$ & $14,915,323$ & $19,376,036$ & $28,000,000$ \\
\hline
\end{tabular}

${ }^{1}$ Excludes disposal, shoreline cleanup, and decontamination costs.

${ }^{2}$ Includes manual shoreline cleanup.

Table 12. Estimated mechanical recovery operation costs for San Francisco Bay crude oil scenarios 1999 U.S. dollars.

\begin{tabular}{crcc}
\hline Spill amount (gal) & $\begin{array}{l}\text { ACP extrapolation } \\
\mathbf{( \$ )}\end{array}$ & $\begin{array}{l}\text { Mechanical recovery } \\
\text { modeling } \mathbf{( \$ )}\end{array}$ & $\begin{array}{c}\text { Etkin historical cost } \\
\text { data model (\$) }\end{array}$ \\
\hline 10,000 & 330,014 & 161,816 & $4,400,000$ \\
100,000 & $1,175,527$ & $1,518,721$ & $22,000,000$ \\
500,000 & $2,856,633$ & $7,537,058$ & $110,000,000$ \\
$5,000,000$ & $10,175,469$ & $24,241,341$ & $181,000,000$ \\
$10,000,000$ & $14,915,323$ & $47,236,798$ & $360,000,000$ \\
\hline
\end{tabular}

${ }^{1}$ Excludes disposal, shoreline cleanup, and decontamination costs.

${ }^{2}$ Includes manual shoreline cleanup.

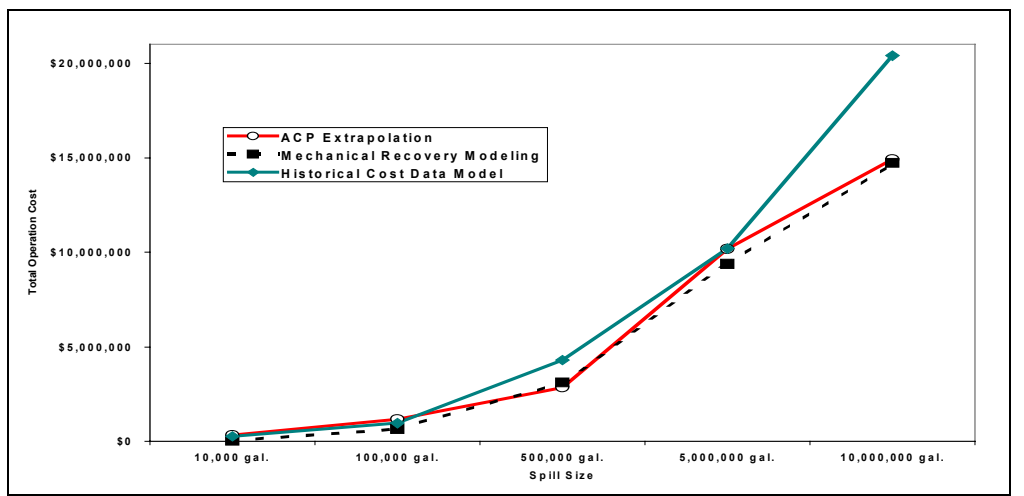

Figure 2. Mechanical recovery operations cost estimations for Texas No. 2 fuel scenario by different methodologies in 1999 U.S. dollars. 


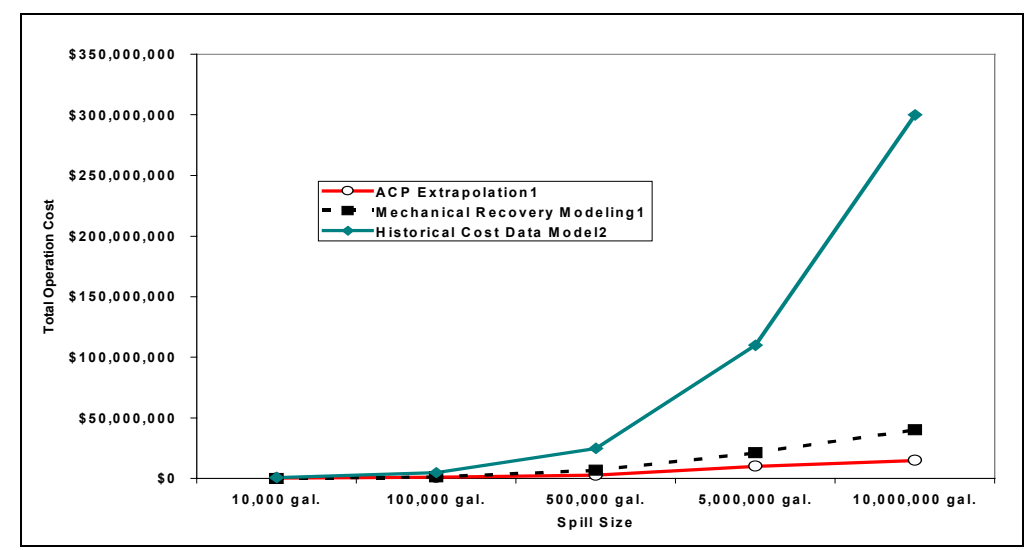

Figure 3. Mechanical recovery operations cost estimations for Texas crude scenario by different methodologies in 1999 U.S. dollars.

\section{Results of dispersant application cost estimations}

Cost estimations for responses involving dispersant use as a primary response strategy from the historical data model (stipulating dispersants as the response method when applying the modifiers) are shown in Figure 4. The costs are roughly 50\% of those estimated for the mechanical recovery operations using the historical data model (from Etkin, 2000).

\section{Mechanical containment and recovery cost estimations}

Since the ACP extrapolation model is based solely on spill amount regardless of oil type and site-specific factors, it does not factor in the evaporation and dispersion rates of a No. 2 fuel as opposed to a crude oil so that there will be different equipment requirements, nor does it factor in that labor and equipment costs differ in California and Texas, for example. The second methodology, which estimates labor and equipment requirements, takes these factors into account and adds in additional costs for federal, state, and spill management costs, and thus is more precise.

The estimates based on the historical model are considerably higher than those of the other methods, particularly for the larger spill sizes. This is due to the fact that the estimation includes the entire response operation, including shoreline cleaning. The costs for the mechanical recovery operations alone would be roughly $15 \%$ of the total costs for spills with significant shoreline impact. Taking this into account, the adjusted estimates based on historical data are relatively close to those of the mechanical recovery modeling method, as shown for the Galveston Bay crude oil scenarios in Figure 5.

This not only supports the general validity of the historical data model, but also suggests that it may be acceptable to use this type of estimation technique when other information is not available or when a rough estimate is required (Figure 6). The model is considerably easier to use than extrapolating information from ACP spill scenarios and the USCG response caps information to develop a hypothetical response strategy to which to apply the estimated equipment and labor requirements to USCG BOA average equipment and labor cost tables.

Since only one methodology was possible for the dispersant cost estimation, it was not possible to test the validity of the historical model for dispersant responses. Historical data and studies on dispersant costs (Etkin 1998a, b, c) suggest that dispersant use can significantly reduce overall and per-unit spill response costs, in large part due to reduced shoreline impact. Cost estimates for dispersant operations using response plan strategies will be further developed in the future in order to compare various cost estimation procedures for dispersant use.

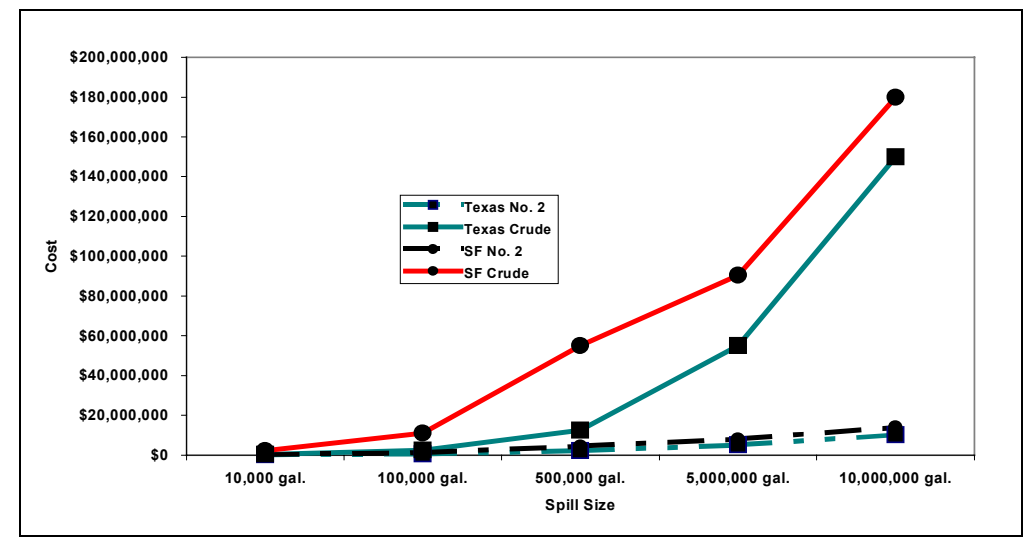

Figure 4. Response operations cost estimations with primary dispersant use (estimated with Etkin historical cost data model) in 1999 U.S. dollars. 


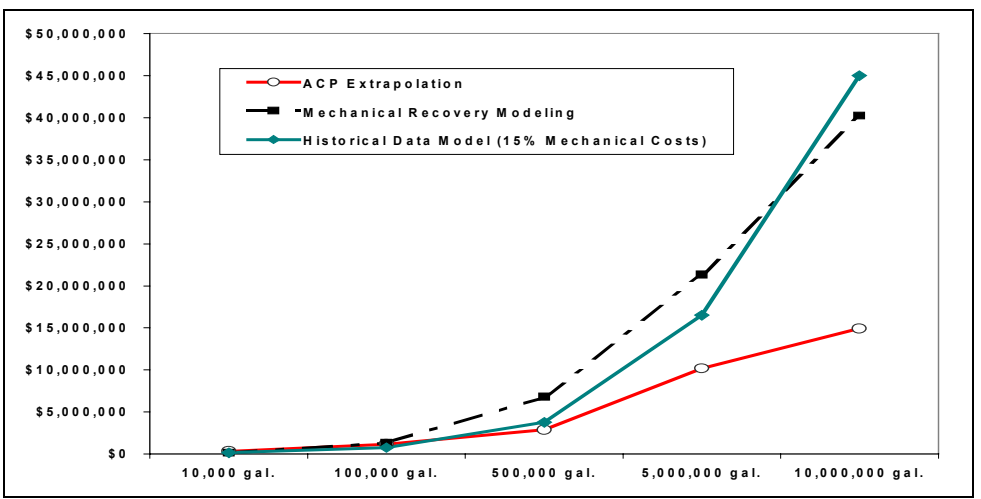

Figure 5. Mechanical recovery operations cost estimations for texas crude scenario by different methodologies, including mechanical-only estimation for Etkin historical cost data model in 1999 U.S. dollars.

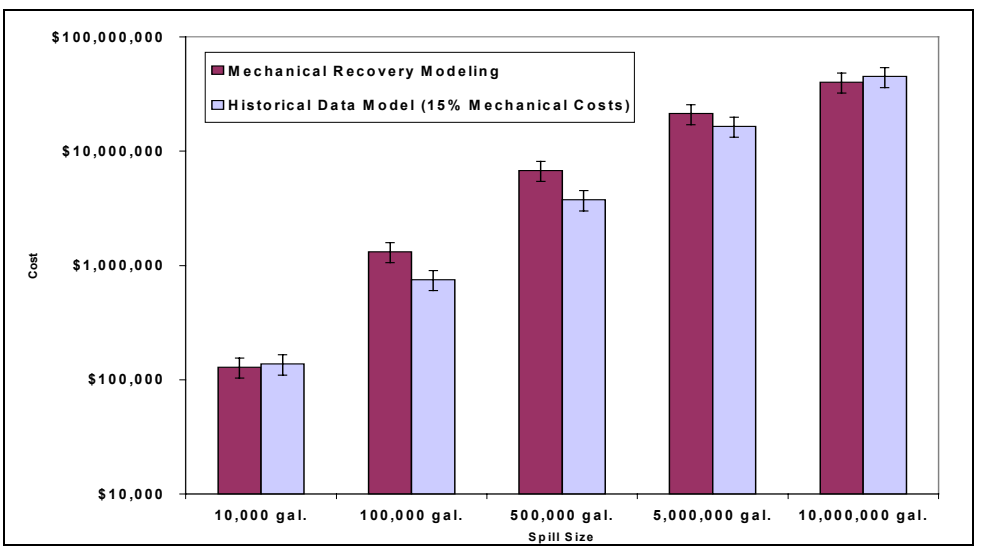

Figure 6. Comparison of mechanical recovery operations cost estimations for mechanical recovery estimation method and Etkin Historical data model for mechanical recovery only (assuming 15\% total costs due to on-water operations) in 1999 U.S. dollars. (25\% error bars.)

The mechanical operations part of a spill response is usually coupled with labor-intensive shoreline cleanup operations, the cost of which can be astronomical. Environmental Research Consulting is conducting further studies on various methodologies for estimating shoreline cleanup costs. These shoreline cost estimation methods will then be linked to mechanical and dispersant operation costs for a more complete picture of oil spill cleanup costs.

\section{Conclusions}

Oil spill response planners, insurers, and other stakeholders would benefit from an oil spill cleanup cost estimation methodology more precise than universal per-unit cost values that have circulated in the industry. The estimation model derived from studies of historical oil spill cost data, as described herein, offers a method for quickly estimating costs for various types of spills based on a number of factors. The comparison to estimates based on hypothetical mechanical recovery-based response plans shows reasonable reliability of the historical database model. Further development and refinement of both the historical cost model and the mechanical recovery model, as well for dispersant operation and shoreline cleanup cost models, will provide even better tools for estimating and predicting oil spill cleanup costs.

\section{Acknowledgements}

A portion of this study was funded under contract to the Transportation Research Board, National Research Council, Washington, D.C.

\section{Biography}

Dagmar Schmidt Etkin received A.M. and Ph.D. degrees from Harvard University. Her environmental science experience includes: 12 years investigating population biology and ecology, and 11 years specializing in oil/chemical spill database development, data analysis, risk assessment, and cost analyses, most recently in her own independent consulting firm, Environmental Research Consulting.

\section{References}

1. Allen, A.A., and R.J. Ferek. 1993. Advantages and Disadvantages of Burning Spilled Oil. Proceedings, 1991 International Oil Spill Conference. American Petroleum Institute, Washington, DC. pp. 765-772.

2. Etkin, D.S. 1998a. Factors in the Dispersant Use Decision-Making Process. Proceedings, Twenty-First Arctic and Marine Oilspill Program (AMOP) Technical Seminar. Environment Canada, Ottawa, Ontario. pp. 281-304. 
3. Etkin, D.S. 1998b. Financial Costs of Oil Spills in the United States. Cutter Information Corp., Arlington, MA.

4. Etkin, D.S. 1998c. Financial Costs of Oil Spills Worldwide. Cutter Information Corp., Arlington, MA.

5. Etkin, D.S. 1999. Estimating Cleanup Costs for Oil Spills. Proceedings, 1999 International Oil Spill Conference. American Petroleum Institute, Washington, DC.

6. Etkin, D.S. 2000. Worldwide Analysis of Oil Spill Cleanup Cost Factors. Proceedings, Twenty-Third Arctic and Marine Oilspill Program (AMOP) Technical Seminar. Environment Canada, Ottawa, Ontario. pp. 161-174.

7. Harper, J., A. Godon, and A. Allen. 1995. Costs Associated with the Cleanup of Marine Oil Spills. Proceedings, 1991 International Oil Spill Conference. American Petroleum Institute, Washington, DC. pp. 167-176.

8. Michel, J., and M. Cotsapas. 1997. Assessment of the Cleanup Costs Resulting From Platform Spills in the Gulf of Mexico Offshore of Apalachicola Bay, Florida. Research Planning, Inc., Columbia, SC.

9. Moller, T.D., H.D. Parker, and J.A. Nichols. 1987. Comparative Costs of Oil Spill Cleanup Techniques. Proceedings, 1987 International Oil Spill Conference. American Petroleum Institute, Washington, DC. pp. 123-127.

10. Monnier, I. 1994. The Costs of Oil Spills After Tanker Accidents. Det Norske Veritas Research A/S, Høvik, Norway.

11. Peck, J., B. Dufour, and V. Peck. 1996. Cost Accounting and Oil Spills. Proceedings, Nineteenth Arctic and Marine Oilspill Program (AMOP) Technical Seminar. Environment Canada, Ottawa, Ontario. pp. 285-314.

12. USCG (U.S. Coast Guard). 1999. Response Plan Equipment Caps Review [draft report]. Office of Response, U.S. Coast Guard Headquarters, Washington, DC, August 1999.

1 Galveston Bay Area Contingency Plan Annex I: Oil Spill Scenarios (1994); Galveston Bay Area Contingency Plan Annex E: Area Assessments (1996); Area Contingency Plan for the California North Coast, San Francisco Bay, and Delta, and Central Coast, Volumes I-III (1997); Philadelphia Area Contingency Plan (1998); Baltimore Area Contingency Plan (1998); and Marine Safety Office Port Arthur Area Contingency Plan [draft] (1999). 